\title{
Síndrome de Fragilidad: Un Desafío para la Venezuela del Siglo XXI
}

\author{
Fragility Syndrome: A Challenge for the Venezuela of the 21st Century
}

\author{
Milagros García \\ Martha2780@gmail.com \\ Universidad Nacional Experimental Francisco de Miranda \\ Venezuela \\ Moreno Martha \\ Martha2780@gmail.com \\ Universidad Nacional Experimental Francisco de Miranda \\ Venezuela
}

Recibido: 15 de noviembre del 2018

Aprobado: 20 de diciembre del 2018

\section{RESUMEN}

La fragilidad es un síndrome multidimensional, con una interacción complicada de elementos biológicos, psicológicos y sociales en el transcurso de la vida individual, que culmina en un estado de mayor vulnerabilidad, asociado a un mayor riesgo de que se provoquen desordenes clínicos opuestos como delirio, deterioro funcional, movilidad perjudicada, caídas, aislamiento social, aumento de la mortalidad. En Venezuela, se determinó que la población de mayor de 65 años representaba el $5,97 \%$, se proyectó para el año 2020 serían el 6,66\% respectivamente de la totalidad de los habitantes. En el año 2025 el $13.23 \%$ de la población de país, tendrá más de 60 años de edad y para el año 2050 será de un $22.10 \%$, se proyecta que la población mayor crecerá $120 \%$ en los próximos años. Es necesaria la planificación innovadora, el desarrollo de políticas de salud sustantivas para mejorar la calidad de vida después de los 65 años de edad.

Descriptores: Síndrome; fragilidad; desafíos; síndrome multidimensional; deterioro funcional. 


\begin{abstract}
Fragility is a multidimensional syndrome, with a complicated interaction of biological, psychological and social elements in the course of individual life, culminating in a state of greater vulnerability, associated with an increased risk of causing opposite clinical disorders such as delirium, functional impairment, impaired mobility, falls, social isolation, increased mortality. In Venezuela, it was determined that the population over 65 years old represented $5.97 \%$, it was projected for the year 2020 would be $6.66 \%$ respectively of the totality of the inhabitants. In $2025,13.23 \%$ of the country's population will be over 60 years of age and by the year 2050 it will be $22.10 \%$, it is projected that the older population will grow $120 \%$ in the coming years. Innovative planning is necessary, the development of substantive health policies to improve the quality of life after 65 years of age.
\end{abstract}

Descriptors: Syndrome; fragility; challenges; multidimensional syndrome; functional deterioration.

\title{
INTRODUCCIÓN
}

Debido al aumento de la esperanza de vida y a la disminución de la tasa de fecundidad, la proporción de personas mayores de 60 años está aumentando más rápidamente que cualquier otro grupo de edad en casi todos los países. ${ }^{1}$ Entre 2015 y 2050, el porcentaje de los habitantes del planeta mayores de 60 años casi se duplicará, pasando del $12 \%$ al $21 \%$ y la mayoría vivirá en países de ingresos bajos y medianos. ${ }^{(2,3)}$

Indudablemente, este cambio poblacional implica un enorme reto para los países, se deben garantizar el disfrute de una buena salud, la cual es fundamental para que las personas mayores mantengan su independencia y puedan tener un lugar en la vida familiar y en su comunidad; prevenir la enfermedad o retrasar la aparición de enfermedades crónicas y no transmisibles, así como su detección y tratamiento oportuno ayudan a reducir al mínimo sus consecuencias. De allí que, el mejor modo de prestar esos servicios es mediante un sistema integral de atención primaria y a través de la gestión de políticas de salud por parte de cada gobierno.( ${ }^{4)}$

Hay que tomar en cuenta que, el envejecimiento de la población y su impacto en los sistemas de ayuda tiende a verse fundamentalmente como un fenómeno, por el cual la 
población en la medida que envejece cada vez más, aumenta igualmente sus demandas sobre todo en atención sanitaria, cuidados y tipo de convivencia; debido a su mayor probabilidad de padecer enfermedades crónicas, tienen un mayor riesgo de discapacidad y por lo tanto, son y serán grandes consumidores de prestaciones sociales y sanitarias tanto formales como informales. A la problemática anterior, se añaden los cambios en la estructura familiar y un debilitamiento de las redes familiares y sociales que está provocando una disminución del cuidado informal y un incremento de las necesidades de cuidados formales y de institucionalización. $(5,6,7)$

A raíz de ello surge, entonces, un cambio de mentalidad: se transforma la medicina curativa hospitalaria en medicina preventiva-asistencial, dado el deseo y la necesidad de añadir calidad a los años vividos, $y$ tomando en cuenta este contexto, surge el concepto de "fragilidad", un síndrome que emerge en los últimos 10 años, que representa el continuo entre un adulto mayor sano y aquel que se encuentra en estado de vulnerabilidad ante estresores externos. Tan sólo una delgada línea separa, en los ancianos frágiles, lo fisiológico y funcional de lo patológico. Una vez traspasada, el camino hacia la discapacidad $y$, posteriormente, a la dependencia puede resultar inminente si no se actúa a tiempo. $\left({ }^{8)}\right.$

Por lo tanto, es un síndrome multidimensional, en donde están involucrados una interacción compleja de factores biológicos, psicológicos y sociales en el transcurso de la vida individual, que culmina en un estado de mayor vulnerabilidad, asociado a un mayor riesgo de que se produzcan desordenes clínicos adversos como delirio, deterioro funcional, movilidad perjudicada, caídas, aislamiento social, aumento de la mortalidad y hospitalización.(9)

En este sentido, el síndrome de Fragilidad debe ser reconocido como objetivo de investigaciones e intervenciones, considerando el impacto que tienen sobre: los individuos ancianos, sus familias y la sociedad; $\left({ }^{10)}\right.$ y a pesar de que en Latinoamérica se vive una transición demográfica y epidemiológica donde las consecuencias de la fragilidad son aún ignoradas y el término es desconocido en los medios no 
especializados; $\left({ }^{11)}\right.$ surge el interés de conocer cuáles son los desafíos que enfrenta la Venezuela del siglo XXI ante ese gran síndrome .

\section{DESARROLLO}

\section{Fragilidad un desafío}

La transición demográfica que viven los países actualmente, hace que estemos ante un reto colosal tanto para los sistemas de salud, la familia y la sociedad: la eclosión epidemiológica de personas con complejidad clínica y necesidades de atención complejas. Frente al incremento de situaciones de complejidad, habitualmente relacionadas con la incertidumbre en el diagnóstico situacional y las dificultades para la toma de decisiones, se necesitarán de nuevos marcos conceptuales y de nuevas herramientas que sean pragmáticas y eficientes. El Síndrome de fragilidad ofrece una excelente oportunidad para revisar y dar a conocer cuáles son los desafíos a lo que se enfrenta nuestra sociedad, también es probable que los índices de fragilidad sean la respuesta más plausible al desafío del diagnóstico de precisión, punto de partida necesario para una atención auténticamente centrada en la persona. (12)

A continuación se presentan cuáles son los desafíos que debe enfrentar la sociedad venezolana ante el síndrome de fragilidad:

\section{1er desafío: Comprender que es el Síndrome de Fragilidad}

El concepto de fragilidad aparece por la necesidad de explicar la heterogeneidad en el proceso de envejecimiento que se observa en la población anciana. Mientras que algunos ancianos llegan a edades avanzadas con una alta reserva funcional ycon capacidad de recuperarse de enfermedades que pudieran surgir, hay otros ancianos cuya reserva vital está agotada, lo cual los hace vulnerables a la enfermedad e incapaces de recuperarse tras la misma. $\left({ }^{13,14)}\right.$

Etimológicamente, el término frágil proviene del francés "frêle", que significa "poco resistente", y del latín "fragilis", que significa "que se rompe con facilidad". A partir de los años 90 el interés se centra en estos nuevos conceptos de fragilidad y anciano frágil, poniéndose de manifiesto la necesidad de identificar a estos pacientes de riesgo 


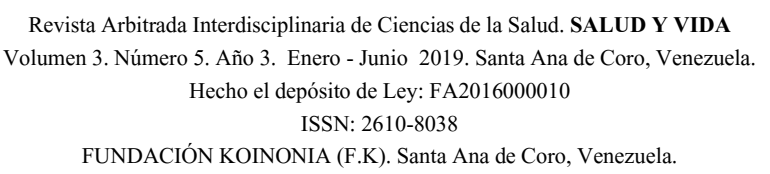

Milagros García; Moreno Martha

que podrían beneficiarse de ciertas intervenciones socio-sanitarias, fundamentalmente preventivas. $\left({ }^{15,16)}\right.$

Aunque no hay todavía una definición ni criterios universalmente reconocidos para su descripción, la fragilidad es un síndrome clínico-biológico caracterizado por una disminución de la resistencia y de las reservas fisiológicas del adulto mayor ante situaciones estresantes, a consecuencia del acumulativo desgaste de los sistemas fisiológicos, causando mayor riesgo de sufrir efectos adversos para la salud como: caídas, discapacidad, hospitalización, institucionalización y muerte. $\left({ }^{17)}\right.$ Esta definición tiene 2 aspectos importantes:

1.Existe una disminución de las reservas fisiológicas en múltiples áreas o dominios, como la resistencia, la flexibilidad y la fuerza muscular, el balance o equilibrio, el tiempo de reacción, coordinación, funcionamiento cardiovascular, audición, visión, nutrición y funcionamiento cognitivo.

2.Existe una predisposición para sufrir efectos adversos para la salud ante situaciones estresantes de la vida cotidiana, donde la magnitud de la situación no siempre está en correspondencia con el menoscabo que origina en la salud, produciéndose gran deterioro funcional. $\left({ }^{18)}\right.$

La búsqueda de una explicación o conceptualización para este síndrome cabe destacar dos Modelos de fragilidad que surgieron al amparo de grandes estudios epidemiológicos enfocados en el envejecimiento poblacional. En primer modelo, surge en base al "Canadian Study of Health andAging" (CSHA, Universidad de Ottawa en 1991), donde Rockwood y Mitnitski proponen el concepto de fragilidad como una acumulación de déficits; $y$ el segundo modelo es el propuesto por Fried y colaboradores (2001), los cuales desarrollan un fenotipo clínico a partir del estudio "Cardiovascular Health Study" (CHS) sobre el cual se asientan las bases de la fragilidad.

A su vez elabora el "ciclo de la fragilidad" que establece que la falta de ejercicio físico, una nutrición inadecuada, la aparición de enfermedades y el exceso de medicamentos, asociados al proceso del envejecimiento, podría dar lugar a una malnutrición crónica y a una pérdida de masas ósea y muscular, produciendo el fenómeno conocido como 
sarcopenia, y a esto, se unen otros procesos como la arterosclerosis, el deterioro cognitivos y las alteraciones metabólicas asociadas, representarían los cuatro mecanismos responsables del origen de la fragilidad $(8,17)$.

Figura №1: Ciclo de Fragilidad

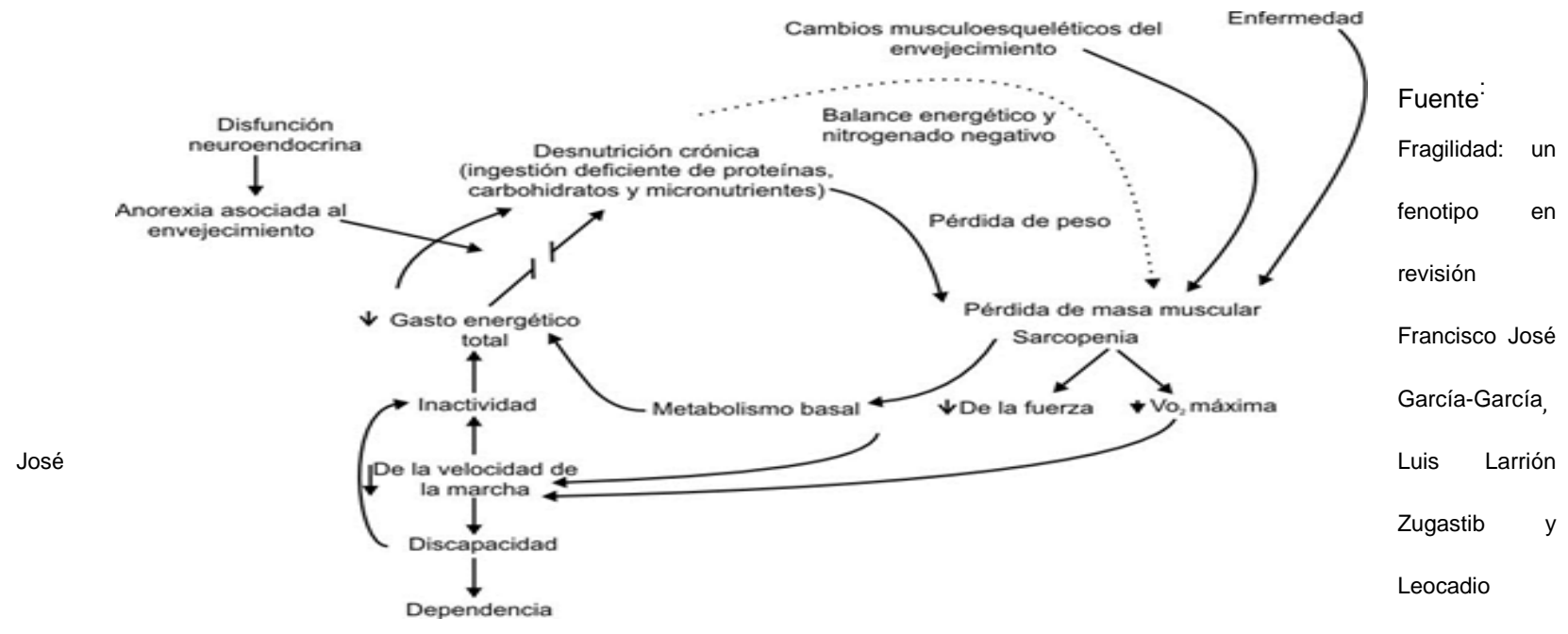

Rodríguez Manas GacSanit. 2011. 25(S): 51-58

Por consiguiente, para este constructo, se identificación con dimensiones, que hacen operativas en cinco criterios, que son utilizados para diagnosticar fragilidad:

1. Disminución de la fuerza muscular.

2. Baja resistencia al esfuerzo o agotamiento autoreferido.

3. Velocidad lenta de lamarcha.

4. Baja actividad física.

5. Pérdida de peso involuntaria de al menos $5 \mathrm{~kg}$ durante el último año. 
De este modo, establecen que aquellos pacientes que cumplan tres o más criterios deberán ser considerados frágiles; si cumplen uno o dos, serán identificados como pacientes prefrágiles y si no cumplen ningún criterio estarán exentos de fragilidad. ${ }^{(8)}$ Esto conlleva a determinar cuáles son los factores de riesgo identificados en el desarrollo de la fragilidad, entre ellos se encuentran: factores biomédicos (presencia de enfermedades crónicas, alteración de la marcha, déficit sensoriales, mala auto percepción de salud, caídas a repetición, polifarmacia, hospitalizaciones frecuentes, institucionalización ), factores funcionales (dependencia en actividades básicas de la vida diaria (ABVD) y dependencia en actividades instrumentales de la vida diaria AIVD)), factores socioeconómicos (vivir solo, viudez reciente, edad $>80$ años, bajos ingresos económicos), factores cognoscitivos/afectivos (depresión, deterioro cognitivo). $(8,19)$

Se hace necesario resaltar, que la fragilidad puede ser buen predictor sobre el riesgo de presentar acontecimiento adversos para la salud, y de causar discapacidad, independientemente de la existencia o no de enfermedades, quizás sea un precursor fisiológico y factor etiológico de la discapacidad, ${ }^{(14,15,16,20)}$ |o que sugiere que los conocidos como síndromes geriátricos, tal vez, sean manifestaciones clínicas de la fragilidad y se puedan considerar, por tanto, indicadores de la misma. ${ }^{(8)}$

En este sentido, las intervenciones dirigidas a frenar el riesgo como serían mantener el normo peso, hacer ejercicio, evitar la inactividad, etc $11,19,{ }^{21}$ han sido efectivas, por ahora, el desafío consiste en comprender que es el síndrome de fragilidad para promover un estilo de vida "sano", en el que se incluya la actividad física y una alimentación equilibrada, a través de programas de promoción a la salud y de programas de prevención, con el propósito de favorecer un envejecimiento óptimo. (11)

\section{2doDesafío: Esta representado por la Epidemiologia y el Síndrome de Fragilidad.} Como se ha mencionado anteriormente, no se conoce cuál es la prevalencia a nivel mundial del síndrome de fragilidad, los estudios epidemiológicos realizados en la última década han avalado los resultados del Cardiovascular HealthStudy (CHS) (2001), que 
reporto una prevalencia de sujetos frágiles del 7\% entre los mayores de 65 años y de prefrágiles del $47 \% .{ }^{22,23}$ Las prevalencias de fragilidad halladas en los estudios con criterios homologables arrojan una estimación del $7 \%$ al 12\%. (24)

La fragilidad es un síndrome frecuente en personas ancianas y que aumenta su prevalencia de manera exponencial a medida que se envejece, desde un $3,2 \%$ a los 65 años, un $16,3 \%$ en los mayores de 80 años y un $23,1 \%$ a los 90 años. Diferentes estudios de cohortes han encontrado prevalencias entre el 4 y el 16,3\% en diferentes ámbitos y países, siendo más prevalente en mujeres y afroamericanos, y con una incidencia a los 75 años del $9 \%$ en mujeres no frágiles. ${ }^{25}$ También conviene destacar, que los estudios españoles, tales como: el Estudio Toledo para un Envejecimiento Saludable (ETES) ${ }^{26}$ arrojo una prevalencia de fragilidad del 8,4\% (mayores de 64 años) y muestra una clara relación con la edad; en el estudio FRADEA ${ }^{25}$ la prevalencia alcanza el 16,9\% (mayores de 69 años); y en el estudio de Peña grande se sitúo en el 10,3\% (mayores de 64 años). ${ }^{(13)}$

No obstante, otros estudios muestran importantes discrepancias en las estimaciones: un estudio realizado en 10 países europeos (Share Study) muestra una prevalencia del $17 \%$, con un $27 \%$ en España. ${ }^{(27)}$

Por su parte, un estudio ealizado en varios países de Latinoamérica y el Caribe, concluyo que la prevalencia en las mujeres osciló entre el $30 \%$ y el $48 \%$, y en los hombres entre el $21 \%$ y el $35 \%$. Es de destacar que, aunque ambos estudios se basan en el constructo de Fried, la operatividad de los criterios es sustancialmente distinta, lo cual nos hace sospechar que estas diferencias pueden explicarse por diferencias metodológicas. ${ }^{(28,14) \text {. }}$

Los estudios de incidencia de fragilidad son más escasos. El Women's Health Initiative Observational Study, con más de 4.000 mujeres, muestra una incidencia de fragilidad a los 3 años de seguimiento del 14,8\%, y el CHS, con más de 5000 hombres y mujeresrefiere una incidencia del $7,2 \%$ en 4 años. La importancia que ha adquirido el síndrome en los últimos años radica en que los sujetos frágiles están en riesgo de importantes eventos adversos sobre la salud a corto, medio y largo plazo. ${ }^{(14)}$ 
Cabe destacar el estudio EPESE realizado en hispanos, muestra que el $84 \%$ de los sujetos clasificados como frágiles fallecieron durante los 10 años de seguimiento, mientras que sólo murieron el 33\% de los no frágiles; relación con la mortalidad que ha sido contrastada en otros estudios. ${ }^{(29)}$ Con base a los estudios realizados en Estados Unidos, se evidencio que el $7 \%$ de los adultos mayores de 65 años son frágiles, aumentando progresivamente con la edad hasta el 25-40\% en mayores de 80 años. Otro estudio realizado en el año 2009 en una comunidad urbana de Cuba, evidenció que el $51.4 \%$ de los estudiados eran frágiles, siendo la polifarmacia el factor de prevalente en un $35.5 \%$ y el sexo femenino tiene un riesgo relativo de ser más frágil con respecto al sexo masculino. ${ }^{(9,30)}$ La fragilidad es un poderoso predictor de discapacidad, hospitalización, caídas, pérdida de la movilidad y enfermedad cardiovascular. ${ }^{(14)}$

En lo referente a las morbilidades asociadas a la fragilidad, se encontró los siguientes: en estudios transversales la alteración cognitiva, el rasgo distímico y la enfermedad vascular presentan una potente asociación con la fragilidad. En estudios longitudinales, estos factores también se muestran como predictores de fragilidad. El estudio ETES también muestra un marcado aumento de la fragilidad para estas afecciones. Desde otro punto de vista, en los sujetos frágiles es casi constante la presencia de estos trastornos: la prevalencia de deterioro cognitivo es del $46 \%$, la de rasgo depresivo del $50 \%$ y la de enfermedad vascular sintomática (isquemia cerebral, cardiopatía isquémica, arteriopatía periférica) del $30 \%$, frente al $9 \%$, el $7,7 \%$ y el $10,7 \%$ en los sujetos no frágiles (datos no publicados del ETES).

La vía causal puede tener varias fuentes, que no son excluyentes entre sí. La fragilidad es una situación inestable, y el tiempo que una persona vive en fragilidad es limitado, lo cual sugiere que puedan compartir bases fisiopatológicas comunes, pero con penetrancia variable ( $p$. ej. mecanismos inflamatorios), que además de favorecer la aparición de enfermedad y de fragilidad predispongan a una persona a entrar en fragilidad una vez avance la enfermedad y predispongan a otra a expresar antes una enfermedad (p. ej., enfermedad de Alzheimer). 
En este sentido, las alteraciones de la pared vascular y de los procesos cognitivosejecutivos-anímicos podrían entrar a formar parte del fenotipo de fragilidad. ${ }^{14} \mathrm{De}$ igual manera, Yabar y colaboradores (2006) en Perú, reportaron una prevalencia de fragilidad de $71.3 \%$ en los adultos mayores. Dentro de las características clínicas de los ancianos frágiles, el $16.1 \%$ presentaba pluripatología siendo frecuentes los síndromes geriátricos agudeza visual y auditiva disminuida (68.4\% y $65 \%$ respectivamente). El $53.5 \%$ mostraba algún grado dependencia, mostró déficit y/o deterioro cognitivo en el $14.9 \%$ y de acuerdo a la Escala de Gijón, en el $77.0 \%$ existía riesgo o problema social. ${ }^{(31)}$

Se han realizado investigaciones acerca de este síndrome en los cuales no se observan diferencias en las variables que resultan asociadas al síndrome de fragilidad: edad avanzada, sexo femenino, bajo nivel educativo, pocos ingresos económicos, comorbilidad, polifarmacia y discapacidad. ${ }^{(9,30)}$ Sin embargo en Venezuela, solo se han realizado hasta los momentos dos estudios relacionado con el síndrome de Fragilidad. El estudio de Moreno y col. (2013) que determinaron la presencia de fragilidad en una comunidad rural del estado Falcón, el cual reporto: $84 \%$ de adultos frágiles (mujeres 46\%), ${ }^{32}$ y el estudio de Bravo A., (2017) realizado en una comunidad urbana del estado Falcón, que reportó una incidencia del síndrome de fragilidad de 4,44\% y $77,77 \%$ fueron catalogados como pre-frágilen adultos mayores pertenecientes a un club de la tercera edad. ${ }^{(33)}$

Es necesario destacar en este renglón, que una de las características del proceso de envejecimiento es el cambio en los patrones de enfermedad y muerte. Las enfermedades crónicas y degenerativas adquieren un papel protagonista y son las más frecuentes entre los mayores en general y entre las mujeres mayores en particular. Las continuas mejoras en el diagnóstico, tratamiento farmacológico y rehabilitador así como en los cuidados han contribuido a reducir la mortalidad asociada a las mismas y favorecido que los mayores con enfermedades reciban mejor atención y tengan un periodo de morbilidad más largo. 
En este sentido se constatan dos realidades: el crecimiento de los mayores de 80 años y la mayor presencia de mujeres en ese grupo poblacional, conforman la mayoría y este fenómeno es conocido como feminización de la vejez. Se ha considerado que en las mujeres mayores se produce una acumulación de desventajas porque viven más años, pero presentan tasas más elevadas de enfermedades crónicas y discapacidad; mantienen un estado de viudez durante más tiempo y en consecuencia tiene más riesgos de soledad.

La generación de mayores actuales confluyen otros aspectos, por motivos culturales sobre ellas ha recaído un trabajo no remunerado, la producción de bienes y servicios (los cuidados en particular) en el ámbito familiar que las hace dependientes de pensiones de viudedad. Estas desventajas, favorecen la presencia del síndrome de fragilidad en este grupo poblacional. Esta realidad requiere nuevos esfuerzos teóricos y prácticos para hacer frente a los desafíos que representan el incremento de estos mayores y su asociación con peor calidad de vida, fragilidad y otras formas de mortalidad psicológica (por ejemplo, la pérdida de la identidad, la autonomía psicológica y el sentido de control). ${ }^{(5)}$

\section{3er desafío: Esta relacionado con la transición demográfica y el síndrome de} fragilidad

Desde el punto de vista demográfico el siglo XXI se inició enfrentando nuevas realidades y nuevos desafíos: el aumento continuado de la población y el incremento en el número de personas de mayor edad. De acuerdo con las proyecciones de las Naciones Unidas, la actual población de 6.6 millardos aumentará hasta 9 millardos en el 2050. Además, la mitad de la población en el 2050 tendrá por lo menos 60 años y para esa época cerca del $80 \%$ de la población mundial mayor de 60 años vivirá en lo que son hoy países en desarrollo. En América Latina en la actualidad, el 9\% de la población tiene 60 años o más, pero en el 2050 uno de cada cinco latinoamericanos tendrá esa edad, y Venezuela forma parte de esa realidad. (7)

El patrón de envejecimiento de la población venezolana sigue un ritmo parecido al de otros países de la región. De acuerdo con información del Instituto Nacional de 
Estadística (INE), Venezuela en cincuenta años cuadruplicó su población, de 5 millones en 1950 hasta alcanzar 26,57 millones en el año y para el año 2011 registró una población de 27.150.095 habitantes. ${ }^{7,34}$ Este crecimiento poblacional fue favorecidos por condiciones políticas, económicas, sociales y culturales, que entre otras cosas provocaron una gran movilidad social y el fortalecimiento de la clase media. ${ }^{(7)}$

En el último censo realizado (2011), el INE señalo que la edad mediana de la población no era de 18 años, sino de 26, lo que coloca al país en una "etapa de transición demográfica", lo que significa que el $50 \%$ de la población se encuentra por debajo de esa edad y el otro $50 \%$ por encima de la misma, lo que apunta a que la población venezolana está envejeciendo. ${ }^{(34,35)}$ De forma específica, para ese mismo año se determinó que la población de mayores de 65 años representaba el 5,97\%, se proyectó que para el año 2020 serían el 6,66\% respectivamente de la totalidad de los habitantes, para el año 2025 el 13.23\% de la población de país tendrá más de 60 años de edad y en el año 2050 será de un $22.10 \%$, se consideró que debía tomarse en cuenta la posibilidad de que algunos pudieran estar en situación de riesgo, ya que con el pasar de los años el estado de salud se deteriora y disminuye la capacidad de autocuidado. ${ }^{(36,37)}$

Es importante señalar, que investigadores en esta área señalan que este proceso de transición demográfica no sigue patrones fijos, sus modalidades están determinadas por las variaciones que experimentan los índices demográficos como la natalidad, la mortalidad y las migraciones, las cuales condicionan la distribución por edades y el crecimiento de la población. Por tal motivo, el éxodo fuera de Venezuela de los últimos tiempos es también un factor a tomar en consideración. ${ }^{(7,35)}$

En este sentido, hay que mencionar, que las pautas migratorias dieron un giro importante enlos años ochenta y noventa, debido a la generalización de un proceso de crisis que restringió la llegada de nuevos flujos migratorios, propició el retorno de los extranjeros que aquí residían y estimuló la emigración calificada de venezolanos al exterior, de jóvenes productivos, que es lo que las sociedades necesita, personas de 20 a 60 años las que han decidido marcharse del país por factores como "la falta de 
oportunidades, el compromiso de su bienestar y el cercenamiento de sus libertades". Si para el año 2011, al menos 5,8\% de la población tenía más de 60 años de edad; "Para este año, seguramente son más personas, porque hay factores que envejecen a la población", planteó el Profesor Iván de la Vega, investigador de la Universidad Simón Bolívar.(38)

Esto trae como consecuencia que el envejecimiento poblacional sea mayor y cuyas consecuencias se harán sentir sobre las variables económicas, el perfil epidemiológico y la estructura social. ${ }^{(39)}$

La vejez venezolana enfrenta múltiples problemas, y entre ellos se encuentra La Fragilidad, la cual va ser el resultante de un Estado omiso y anacrónico en políticas gerontológicas y geriátricas. El resultado es la ausencia casi total de estructuras destinadas al bienestar integral del adulto mayor y una seguridad social para la vejez en crisis permanente. ${ }^{(40)}$

Este hecho es relevante, ya que a mayor número de adultos mayores, mayor será el número de personas frágiles, ya que el síndrome de Fragilidad, es una entidad clínica relacionada con la edad, es un indicador fundamentar para mejorar la calidad de vida del adulto mayor, por lo que su detección y diagnóstico temprano es fundamental. Sin embargo, ofrecer una valoración de la fragilidad es una tarea complicada, debido a la cantidad de los factores que deben tenerse en cuenta para establecer un diagnóstico integral, por lo que se considera un área de especial relevancia en diversos sectores de la comunidad científica, que buscan ofrecer mecanismos que faciliten su análisis y valoración lo que representa uno de los principales desafíos que en la actualidad enfrentan los profesionales de la salud, en especial los que se dedican a la atención primaria. ${ }^{(30,41,42,43)}$

\section{4to desafío: Se refiere a las políticas de salud y fragilidad}

El fenómeno del envejecimiento de la población, iniciado en los países desarrollados hace varias décadas, avanza con rapidez, impulsando a las organizaciones internacionales, a los gobiernos, asociaciones científicas y gremiales, a analizar y 
plantearse políticas públicas en materia de seguridad social, cuya acción esté dirigida a la promoción de servicios sociales y protección social (programas de salud, vivienda, recreación, educación, capacitación y otros) que den respuestas oportunas, adecuadas y económicamente factibles a los efectos ocasionados por el envejecimiento. ${ }^{(44)}$ Indudablemente, el desarrollo de políticas de salud dirigidas a la población de adultos mayores comparte retos y desafíos con el resto de las políticas de salud que abarcan a toda la población, o a otros sectores. Es el caso de la importancia de disminuir las inequidades, velar por la garantía de derechos y otros múltiples aspectos. Efectivamente, los retos asociados al envejecimiento no pueden considerarse aislados de otras categorías de la población a efecto tanto de análisis de las situaciones como del diseño e implementación de políticas. Existen distintas dimensiones de envejecimiento de acuerdo al género, etnia, y clase social que deben considerarse a la hora de planificar y desarrollar dichas políticas. ${ }^{(45)}$

En Venezuela, se han hechos aportes importantes orientados hacia la protección del adulto mayor en cuanto seguridad social (pago de pensiones y bono de alimentación) se refiere, pero aún existen muchos aspectos de los cuales no se ha adelantado en relación a mejorar su calidad de vida, tal es el caso de las políticas de salud, las mismas, no se encuentran adaptadas al contexto geriátrico de salud y calidad de vida en adultos mayores institucionalizados, donde los resultados obtenidos reflejan que las condiciones no son las adecuadas para su bienestar físico, mental y emocional.. ${ }^{(46)}$

En relación a los programas de atención primaria en salud para el adulto mayor, se determinó que no se han desarrollado plenamente, por lo que se debe tomar en cuenta que dicho proceso está asociado a una disminución en la ejecución de actividades vitales y potenciales aunado a un incremento de la susceptibilidad a enfermar o morir; esto lo señalan los estudios realizados con respecto al envejecimiento, donde sobresale que la vulnerabilidad originada con la edad viene de la mano con los estilos de vida poco saludables, mal funcionamiento, poca adaptación del organismo al medio ambiente, y pobre apoyo familiar. ${ }^{(46)}$ 
Los desafíos del envejecimiento de la población y de los adultos frágiles son globales, nacionales y locales, satisfacer estos desafíos requerirá de una planificación innovadora y de desarrollo de políticas de salud publicas sustantivas que favorezca a este gran número de personas. Entre estos desafíos se pueden mencionar los siguientes:

1.- El crecimiento demográfico de la ancianidad (el siglo XXI puede ser llamado el siglo del envejecimiento), represente una novedad con respecto a épocas anteriores, significando un peso notable sobre la sociedad que lo debe aceptar con espíritu de solidaridad, obligando a realizar ajustes sociales y repercutirán en el ámbito médico.

2.- En las próximas décadas nuestro país completará la transición demográfica. Ocurrirán grandes cambios al interior de las familias y dará lugar a cambios significativos en los patrones de consumo.

3.-Generará una mayor necesidad de medicamentos para patologías crónicas, para la atención de los diferentes síndromes geriátricos incluyendo la fragilidad, lo que originara fuertes presiones a los sistemas de seguridad social. ${ }^{(47)}$

4.- La disminución del número de personas que componen la población activa y el envejecimiento progresivo de esa población producirán desequilibrios que obligarán a cambios en la política de jubilación. Es necesario que el Estado o los gobiernos se planifiquen adecuadamente para afrontar los desafíos planteados a los sistemas de la seguridad social y de pensiones. El objetivo debe consistir en reconocer y aprovechar el talento y la experiencia de las personas mayores y garantizarles un nivel de vida adecuado, a la vez que se fomentan los intercambios armoniosos entre generaciones. ${ }^{(48)}$

5.-Los desequilibrios en la inversión y en el ahorro a nivel colectivo y familiar contribuirán a una posible disminución en las rentas familiares disponibles.

6.- Los desequilibrios causados en las estructuras familiares, serán determinantes del aumento de la problemática de socialización intergeneracional.

7.- Se ejercerán fuertes presiones sobre la infraestructura de salud y se insistirá en la reasignación de recursos para la atención de enfermedades crónico degenerativas. El 
aumento del gasto sanitario por discapacidades conducirá a profundas reformas en las estrategias, alcance, funcionamiento y organización de este sector. $\left({ }^{47)}\right.$

8.- Doble carga de la enfermedad: los países en vía de desarrollo continúan luchando contra las enfermedades infecciosas, la desnutrición y las complicaciones perinatales, también se enfrentan con el rápido crecimiento de enfermedades no transmisibles (ENT). Esta «doble carga de la enfermedad» lleva los escasos recursos al límite, donde las enfermedades crónicas como las enfermedades cardíacas, el cáncer y la depresión, están convirtiéndose con rapidez en las causas principales de morbilidad y discapacidad.

9.- Mayor riesgo de discapacidad, lo que resulta costoso y reducen la calidad de vida. Las discapacidades físicas o mentales hacen que le resulte difícil llevar a cabo las actividades de la vida diaria; la probabilidad de experimentar estas graves discapacidades aumenta de forma espectacular en edades muy avanzadas. Es significativo que los adultos de más de 80 años son el grupo de edad de más rápido crecimiento en todo el mundo, por lo que representa el grupo de mayor riesgo.

10.- Proporcionar asistencia a las poblaciones que envejecen, uno de los mayores desafíos de la política sanitaria es encontrar el equilibrio entre la ayuda para el cuidado de la propia salud (personas que se cuidan a sí mismas), la ayuda informal (asistencia de los miembros de la familia y amigos) y la atención formal (servicios sociales y de salud). La atención formal incluye tanto la asistencia primaria de salud (prestada básicamente al nivel de la comunidad) como la asistencia institucional (en hospitales o residencias de ancianos).

11.- Las acciones de promoción de la salud y de prevención deberán desempeñar un papel cada vez más preponderante y emprenderse desde muy temprano en la vida de las personas, con el objeto, no sólo de promover la salud en el adulto mayor, sino de alcanzar la meta de mejorar la calidad de vida, junto con la prevención de la discapacidad y de la fragilidad, lo cual exigirá la elaboración de propuestas legislativas realistas y adecuadas a la nueva situación social. Parte de esta disminución se debe 
probablemente a los mayores niveles de educación, a los mejores estándares de vida y a una mejoría de la salud en los primeros años de la vida.

12. Forjar un nuevo paradigma: Tradicionalmente, la vejez se ha relacionado con la enfermedad, la dependencia y la jubilación. Las políticas y los programas vinculados a este paradigma tradicional están desfasado no reflejan la realidad, ya que los paradigmas emergentes (el envejecimiento activo y productivo), están relacionados con la autonomía y el empoderamiento del adulto mayor. Ha llegado el momento de instaurar un nuevo paradigma que considere a las personas mayores participantes activas de una sociedad que integra el envejecimiento y que considere a dichas personas contribuyentes activos, beneficiarios del desarrollo, donde incluya personas mayores que están enfermas, frágiles y vulnerables, que permitan la defensa de sus derechos de asistencia y seguridad.

Es necesario un enfoque intergeneracional, que reconoce la importancia de las relaciones familiares y el apoyo que se prestan entre sí los miembros y las generaciones en una familia. Donde los propios ancianos y los medios de comunicación tomen la iniciativa para forjar una imagen nueva y más positiva del envejecimiento y sobre todo del adulto pre frágil y frágil. El reconocimiento político y social de las contribuciones que las personas de edad hacen y la inclusión de hombres y mujeres de edad en los papeles directivos apoyarán esta nueva imagen y ayudarán a deshacer los estereotipos negativos. Educar a los jóvenes con respecto al envejecimiento y prestar una cuidadosa atención al mantenimiento de los derechos de las personas de edad ayudará a reducir y eliminar la discriminación y el abuso.(48)

En Venezuela aún está dando sus primeros pasos en adecuar las condiciones de salud, ambiente y comunidad, hay un gran avances a nivel de políticas públicas, pero es necesario trabajar en educación para la salud en las comunidades, donde se conciencie a la colectividad sobre la importancia del cuidado del anciano en el hogar, por lo cual se puede avizorar la poca existencia de un programa efectivo hacia el enfrentamiento de este fenómeno en la población que contemple además un diagnostico epidemiológico, psicológico, comunitario, y clínico; ya que para el Estado los problemas básicos 
sanitarios relevantes son la desnutrición, enfermedades epidémicas, educación muy reducida a conocimientos necesarios para la mejora de la calidad de vida y con una economía en declive, originando que ésta realidad merme los esfuerzos para el logro de las necesidades sociales en este grupo de edad.(.46)

\section{5to desafío: Es el concerniente a la economía, los costos en salud y de la familia.}

Estos cambios demográficos que ya nos están llegando, imponen grandes desafíos a los gobiernos de nuestros países, los cuales deben promover medidas y acciones oportunas para atender a una población que envejece, factor que compromete seriamente el desarrollo de la región. La OMS ha señalado que el envejecimiento de la población afectará tanto al mundo en desarrollo como al desarrollado. Pero "los países en desarrollo envejecerán antes de volverse ricos. Además de asignar un PIB adecuado para atender a los adultos mayores, es necesario ayudar a crear una cultura en salud y para la salud, la cual debe iniciarse en la juventud.

En este sentido, llama la atención, el debilitamiento económico de la clase media, la cual, de acuerdo con ese organismo se convierte en factor negativo para el desarrollo. Y es que no será la clase media la única que sufrirá las consecuencias del deterioro de las economías de la región, serán todos los grupos sociales menos pudientes los que pagarán la cuota más alta de los efectos de una mala nutrición, de enfermedades relacionadas con los cambios climáticos y las carencias de servicios sanitarios. Pero las poblaciones más vulnerables serán los niños, los ancianos y los enfermos. $\left({ }^{7)}\right.$

El envejecimiento poblacional está caracterizado por el aumento en los gastos de salud destinados al manejo de padecimientos crónicos como las enfermedades cardiovasculares, diabetes mellitus, enfermedad de Alzheimer, enfermedades oncológicas, enfermedad pulmonar obstructiva crónica (EPOC) y problemas músculo esqueléticos. Los sistemas de salud serán desbordados por estos problemas. Los actos médicos de detección precoz, intervención apropiada, manejo y seguimiento de estas enfermedades crónicas tienen lugar preponderante en el ámbito de la atención primaria. La OMS considera que los Centros de Atención Primaria son el mecanismo ideal para 
ofrecer el cuidado de los gerontes a nivel de la comunidad. Sobre este problema la Dirección General de Enfermedades no Reportables y Salud Mental dice: "Ayudar a la gente mayor a permanecer saludables y asegurar una buena calidad de vida en sus últimos días es uno de los desafíos más grandes del sector de salud en todos los países"....". Descuidar la atención de los adultos mayores con enfermedades crónicas, aumentará su incidencia, prevalencia y complicaciones. Además, el incremento de costos, restringirá recursos para áreas prioritarias como la salud materno-infantil.

A las penurias del envejecimiento se suman los efectos nocivos de un mercado de los medicamentos cada vez más excluyente, que además de sus altos costos, se incrementa por la tendencia a la "polifarmacia, suma de inexperiencia con mala formación de muchos profesionales de la medicina, elevando aún más el costo de la cuota diaria requerida para el mantenimiento de la salud en los adultos mayores, ya que numerosos estudios han demostrado que las personas mayores de 65 años toman un promedio de 5 a 7 medicamentos, casi todos innecesarios. ${ }^{7}$

Tal como se ha venido señalando anteriormente, un adulto frágil es una persona dependiente y discapacitado (funcional o psíquico) por lo que amerita de un cuidador o de un familiar, por lo tanto no es un ente productivo ni para el estado ni para su familia, por lo que el estado debe garantizar su seguridad y protección social, además de servicios de salud oportuno; ya que tienen mayor predisposición a hospitalización, lo que representa un aumento del gasto público, aumento de la estaría hospitalaria, tiene varias comorbilidades asociadas que requieren de varios y diversos medicamentos, lo que genera aumento en las morbilidades y en la mortalidad. Como resultado, esto producirá una acumulación de costos para el individuo, la familia y el estado a medida que aumenta la edad y el número de personas frágiles. (46)

Pero en realidad, esta entidad nosológica, que se presenta en los adultos mayores no es detectada y atendida oportunamente, lo que genera dependencia total, afectando la calidad de vida del paciente que requerirá de mayores recursos, como se mencionó anteriormente ( familiares, sociales y en este escenario a las instituciones responsables de su atención), poniendo a los sistemas de salud de países en desarrollo 
en alerta y en la necesidad de reestructurar sus servicios y establecer medidas preventivas para la atención a la salud.

En consecuencia al calcular los costos en base al perfil de uso de pacientes con síndrome de pre-fragilidad y fragilidad, estos no muestran gran diferencia en la inversión de recursos económicos porque su manejo es similar al indicado en enfermedades crónico degenerativas dejando de lado la prevención y manejo de esta situación de salud propia de los ancianos.

Cuando se analiza el manejo y tratamiento preventivo y de control que se lleva en las enfermedades crónicas, se identifica que las acciones preventivas que deberían llevarse a cabo en el manejo del síndrome de pre-fragilidad y fragilidad se basan en el ejercicio y la dieta, se podría pensar que este se atiende de manera indirecta. Siguiendo con el mismo razonamiento, entonces, la población adulta mayor que no las padece pero ya presenta este síndrome por el propio envejecimiento, se abre como área de oportunidad para el manejo preventivo.

El costo en salud se relaciona al tratamiento de enfermedades crónicas y no a los síndromes de pre y fragilidad que, como entidad propia debieran tratarse oportunamente en pacientes con o sin presencia de enfermedad crónico degenerativa.(49)

Por lo tanto, la fragilidad debe ser una prioridad en el desarrollo de las políticas de atención sanitaria de los adultos mayores. Se hacen necesaria la identificación del grupo de mayores frágiles porque esto permitirá implementar actuaciones preventivas y terapéuticas que retrasen o reduzcan la carga de discapacidad y dependencia, debe ser una prioridad en la elaboración de las políticas de atención sanitaria a los mayores. Las principales intervenciones están en prevenir e incluso revertir el estado de fragilidad, a través del ejercicio físico (posible y necesario a cualquier edad monitorizado por expertos), una nutrición ajustada a las necesidades específicas de los mayores, la valoración geriátrica y la intervención sobre los principales síndromes geriátricos con un ajuste de la medicación a las características de esta población. Asimismo, están apareciendo en los últimos años modelos de atención sanitaria 
específicos coordinados entre atención primaria y especializada para identificar y tratar a los adultos mayores.

Por lo anteriormente mencionado, el desafío esta en desarrollar una cultura de sociedad moderna, no enfocada en paliar las consecuencias del paso de los años, sino a promover un envejecimiento saludable y la educación integral de todos sus componentes, contribuyendo a no medicalizar tanto esta etapa de la vida, que puede ser de disfrute y satisfacción sin uno de sus grandes fantasmas llamado la fragilidad. $(46,50,51)$

\section{6to desafío: Está relacionado con la familia}

El envejecimiento demográfico ha ido acompañado de una creciente seguridad en la supervivencia de los miembros de la familia hasta bien entrada su vejez, lo que ha ocasionado cambios en la estructura familiar, permitiendo visualizar cuatro diferentes situaciones en torno al anciano:

1- los que viven solos,

2- los que residen con el cónyuge, más hijos y parientes,

3- los que viven con hijos y parientes y sin el cónyuge, y

4- aquellos que viven apenas con el cónyuge.

Las alteraciones en los estándares de mortalidad y fecundidad introdujeron modificaciones importantes en la arquitectura de las familias que pueden influenciar en la forma como cuidan a sus ancianos. La disminución de la fecundidad redujo el tamaño de las familias, resultando en una disminución de las relaciones entre generaciones y la longevidad, a su vez, trajo el aumento de familias multigeneracionales a pesar de esta situación, no hay garantía de que, las familias estén preparadas para asumir el papel de cuidadoras del anciano. ${ }^{(52,53)}$

Las principales limitaciones frente a la necesidad de cuidar a un anciano, en el contexto familiar, están relacionadas a los aspectos de orden financiera, personal y social. Cuando surgen situaciones de fragilidad y dependencia, hay necesidad de realizar 
adaptaciones y de reorganizar a la familia, lo que se refleja en el ajuste de los roles familiares, definidos a lo largo del tiempo y de las formas como cada miembro interactúa con los demás. Al mismo tiempo, reajustes en la estructura familiar dependen de como los cambios y eventos se originaron y de los recursos disponibles para lidiar con esas modificaciones. ${ }^{(52)}$

Además, se considera que las transformaciones familiares (menor número de hijos promedio, mayor número de mujeres casadas o unidas incorporadas al mercado laboral, salarios que impiden enfrentar el sostenimiento de un mayor número de dependientes en la familia y mayor cantidad de adultos mayores que atender por familia) el apoyo y cuidado del adulto mayor se advierte más complicado en las próximas décadas, pese a que la familia en el momento actual sigue realizando un gran esfuerzo por dar respuesta a las necesidades del adulto mayor, particularmente de las mujeres (madres cuando envejecen), puesto que en el varón lo más común es que reciba las atenciones y cuidados necesarios de su cónyuge (generalmente menor).

Entender el proceso de envejecimiento enfrentado por el adulto mayor y especialmente si es frágil no es sencillo, se trata de situaciones complejas y heterogéneas en las que influyen no solo las diferencias de género y el incremento de la edad, sino también las situaciones socio-familiares y el estado anímico del adulto mayor, así como los recursos con los que cuenta observados en el nivel educativo que se tiene y el estrato socioeconómico al que se pertenece. Pero además, se hace necesario entenderla manera en que surge una discapacidad o fragilidad en el adulto mayor y particularmente, la edad en la que se presenta, permitiendo identificar el alcance y retos a enfrentar tanto por el adulto mayor, como por su familia y la sociedad en general, que soportarán las necesidades y dependencia del adulto mayor, lo que representa una sobrecarga social y económica que es necesario prever desde todos los ámbitos. ${ }^{(54)}$ El apoyo y el acompañamiento que las familias brindan a sus mayores, se ve fragmentado ante la presencia de enfermedades mentales, neurológicas o físicas, que forman parte de los síndromes geriátricos, (entre ellos el de fragilidad) que los tornan dependientes. Esto genera agotamiento de los cuidadores, hasta el punto de tener 
familias completas enfermas por falta de ayuda, capacitación y reconocimiento, Lo grave es que todo esto llega fácilmente al maltrato, una tendencia más preocupante que la institucionalización y, en muchos casos, hacia el abandono. El aumento de las hospitalizaciones de adultos mayores sin acompañantes en épocas de vacaciones es prueba de esto. ${ }^{(53)}$

Como se ha mencionado con anterioridad, los cambios, en los modelos de la familia, asociados al aumento del segmento de la población con creciente grado de fragilidad, dependencia y discapacidad, producen un fuerte impacto en las familias tanto desde punto de vista emocional como del financiero. ${ }^{(53)}$

Otro aspecto importante a considerar es la funcionalidad familiar, la cual es dinámica, ya que las familias no son estáticas y por lapsos o circunstancias pueden caer en crisis, $y$ en ese momento ser disfuncionales en mayor o menor grado, y recuperarse posteriormente. Hay que tomar en cuenta que la enfermedad o fragilidad de un adulto mayor que le ocasione dependencia y discapacidad puede generar en algún momento una crisis familiar, así como, el agotamiento del cuidador, ya que algunas familias ante estos problemas no consiguen su homeostasis y la disfunción se vuelve irreparable o llegan a la desintegración, no obstante el mayor porcentaje de familias recuperan su funcionalidad sin ayuda técnica específica.

Los incidentes afectan a los miembros de una familia en forma distinta en diferentes momentos, por eso, la homeostasis familiar puede funcionar adecuadamente para ellos durante algunos periodos de la vida familiar y no durante otros. El mantenimiento de éste equilibrio es un requisito indispensable para que la familia cumpla con sus funciones eficazmente. Una familia con una dinámica funcional mantiene un estado de equilibrio $u$ homeostasis tal, que las fuerzas del grupo le permiten progresar de crisis en crisis, resolviéndolas de manera que se promueve el desarrollo de cada miembro hacia un mayor grado de madurez. ${ }^{(55)}$

Finalmente, otro aspecto a tratar es la convivencia y estructura familiar. Los individuos envejecen en la actualidad de forma muy diferente a como lo hacían sus antepasados, tanto en lo que atañe a las formas de convivencia (respecto a las cuales han 
protagonizado una verdadera emancipación), como a la coexistencia intergeneracional, a las relaciones familiares consecuentes, a su papel dentro de la familia y a la respuesta que ésta puede dar cuando aparecen las situaciones de dependencia, más frecuentes ahora que en generaciones anteriores.

Se observa, aunque de forma incipiente y con rasgos contradictorios, que los mayores consideran la vida autónoma, en su propio hogar un valor en sí mismo. El aumento de la autonomía residencial es consecuencia de las mejoras en las condiciones de salud y de la independencia económica. Debido a la menor esperanza de vida de los hombres, las mujeres viudas son más frecuentes, viven más tiempo en hogares solitarios o unipersonales y por tanto su riesgo de sufrir soledad es mayor, cuando comienzan los problemas de dependencia y de necesidad de ayuda, además de experimentar aislamiento social y de privación económica. Lo que los haces más susceptible a desarrollar el síndrome de fragilidad.

La tarea del cuidado sigue recayendo en la familia y dentro de ella en las mujeres como cuidadores principales. Aunque se observa un cambio en la tendencia con una mayor presencia de la ayuda formal, a través de centros de día, ayuda a domicilio y mayor oferta de institucionalización y esto es debido la incorporación de la mujer al mercado laboral y las migraciones de los hijos, entre otros motivos, lo que propician que haya menos disponibilidad familiar para el cuidado de los más mayores e implica nuevas necesidades de servicios profesionales y de cuidados formales que apoyen o suplan a los cuidadores familiares.

Recientemente se ha empezado a valorar realmente y a incorporar a las políticas públicas la importancia de los cuidados informales reconociendo, mediante prestaciones monetarias y técnicas, a los cuidadores que se ocupan de los mayores en el ámbito familiar o fomentando ayudas a domicilio, unidades de respiro entre otros. Este sistema, conjuga cuidados formales e informales, permite evitar o retrasar la institucionalización, en algunos casos. Aunque se temía que el hecho de proporcionar servicios de asistencia profesionalizada podría conllevar una menor implicación por parte de la familia, los estudios muestran que cuando la responsabilidad del cuidado 
recae exclusivamente en ella, es cuando se observa un claro retroceso en su papel cuidador que suele desembocaren ingreso en una institución, unas veces porque son incapaces de asumir esta tarea en exclusividad, otras porque se agotan ante la situación de sobrecarga que comporta. Esto es una buena muestra de la voluntad de complementar las responsabilidades públicas y familiares en este complejo proceso. $\left(^{(5)}\right.$

\section{CONSIDERACIONES FINALES}

-El síndrome de fragilidad es un indicio clínico-biológico caracterizado por una disminución de la resistencia y de las reservas fisiológicas del adulto mayor ante situaciones estresantes, a consecuencia del acumulativo desgaste de los sistemas fisiológicos, causando mayor riesgo de sufrir efectos adversos para la Salud.

- En Venezuela se proyecta que la población anciana crecerá aún más en los próximos años, por lo que es necesario comenzar a preparar un plan nacional de envejecimiento, para la prevención de enfermedades y para mejorar la calidad de vida de las personas después de los 65 años de edad.

-Se hace necesario que las acciones de promoción de la salud y de prevención desempeñen un papel cada vez más preponderante y emprenderse desde muy temprano en la vida de las personas, con el objeto, no sólo de promover la salud en el adulto mayor, sino de alcanzar la meta de mejorar la calidad de vida, lo cual exigirá la elaboración de propuestas legislativas realistas y adecuadas a la nueva situación social. - Se considera que aportar asistencia a las poblaciones que envejecen, es uno de los mayores desafíos de la política sanitaria es encontrar el equilibrio entre la ayuda para el cuidado de la propia salud (personas que se cuidan a sí mismas), la ayuda informal (asistencia de los miembros de la familia y amigos) y la atención formal (servicios sociales y de salud). La atención formal incluye tanto la asistencia primaria de salud (prestada básicamente al nivel de la comunidad) como la asistencia institucional (en hospitales o residencias de ancianos). 
-Se cree que las inestabilidades causadas en las estructuras familiares, serán determinantes del aumento de la problemática de socialización intergeneracional del síndrome de fragilidad en el siglo XXI.

- La fragilidad debe ser una antelación en el perfeccionamiento de las políticas de atención sanitaria de los adultos mayores. Se hacen necesaria la identificación del grupo de mayores frágiles porque esto permitirá implementar actuaciones preventivas y terapéuticas que demoren o reduzcan la carga de discapacidad y dependencia.

-Es necesario desarrollar una cultura de sociedad moderna, no enfocada en paliar las consecuencias del paso de los años, sino a promover un envejecimiento saludable y la educación integral de todos sus componentes.

\section{REFERNECIAS CONSULTADAS}

1. OMS. (2018) Envejecimiento. Disponible en: http://www.who.int/topics/ageing/es/

2. OMS. (2018). Envejecimiento y salud. Disponible en: http://www.who.int/es/news-room/fact-sheets/detail/envejecimiento-y-salud.

3. Cardona Arangoa, Doris; Segura Cardona, Ángela María. (2011). Políticas de salud pública aplicadas al adulto mayor en Colombia. Revista española de geriatría y gerontología Vol. 46. Núm. 2.Marzo - Abril 2011. Páginas 59-116 Disponible en: http://www.elsevier.es/es-revista-revista-espanola-geriatriagerontologia-124-articulo-politicas-salud-publica-aplicadas-al$\underline{\text { S0211139X10002490 }}$

4. (2015) ¿Qué repercusiones tiene el envejecimiento mundial en la salud pública? Disponible en: http://www.who.int/features/qa/42/es/

5. Garrido A., Margarita (2012) Longevidad y cuidados de larga duración en España: Diferencias en la calidad de vida relacionada con la salud y en la fragilidad entre hombres y mujeres institucionalizados. Tesis doctoral. Universidad de Castilla-La Mancha.

6. HeungBongCha. La misión de la IAGG respecto a la fragilidad en el anciano. Libro blanco de la Fragilidad. Disponible en:http://www.semeg.es/uploads/archivos/LIBRO-BLANCO-SOBREFRAGILIDAD.pdf 
7. Osuna C.,Jesús A. (2009) Globalización y envejecimiento. Rev. Venez. Endocrinol. Metab. v.7 n.1 Mérida feb. 2009. Disponible en: http://www.scielo.org.ve/scielo.php?script=sci arttext\&pid=S1690$\underline{31102009000100002}$

8. Rojo Noriega Elena (2016). "Síndrome de fragilidad: detección y abordaje" Trabajo de fin de grado para enfermería Universidad de Cantabria. Disponible en:

https://repositorio.unican.es/xmlui/bitstream/handle/10902/8929/Rojo\%20Noriega \%20E..pdf?sequence $=4$

9. Ramos O.,Daniela, BettinelliLuiz A,Pasqualotti Adriano,Corso Débora, BrockAlacoqueFelipe, ErdmannLorenzini.(2013). Prevalencia de síndrome de fragilidad en los adultos mayores de una institución hospitalaria Rev. Latino-Am. Enfermagem 21(4): [08 pantallas] jul.-ago. 2013 Disponible en: www.eerp.usp.br/rlae

10.Sousa P., Maycon, Dos Santos T., Darlene M. (2014). Factores asociados al síndrome de fragilidad en ancianos residentes en área urbana. Rev. Latino-Am. Enfermagem sept.-oct. 2014; 22(5):874-82 Universidad Federal do TriânguloMineiro, Uberaba, MG, Brasil. Disponible en: http://www.scielo.br/pdf/rlae/v22n5/es 0104-1169-rlae-22-05-00874.pdf (consultado el 12/10/18)

11.Ávila-Funes, José Alberto, Aguilar-Navarroa Sara, Melano-Carranza, Efrén. (2008). La fragilidad, concepto enigmático y controvertido de la geriatría. La visión biológica. GacMédMéx Vol. 144 No. 3, 2008 Disponible en: http://www.medigraphic.com/pdfs/gaceta/gm-2008/gm083j.pdf

12. Amblàs-NovellasaJordi,Espaulella-PanicotbJoan,Inzitarid Marco,RexachfLourdes, Fontechag Benito, Romero-rtunohRoman. (2017).En busca de respuestas al reto de la complejidad clínica en el siglo xxi: a propósito de los índices de fragilidad. Revista española de Geriatría y Gerontología. Vol 52. Num 3, mayo - junio 2017 Disponible en: http://www.elsevier.es/es-revista-revista-espanola-geriatriagerontologia-124-articulo-en-busca-respuestas-al-reto-S0211139X16301044

13. Castillo GallegoCarmen. (2015). Influencia de la masa corporal y dela distribución grasa sobre la fuerza muscular de una cohorte de población anciana: Diferencias por sexo. Tesis Doctoral. Madrid 2015.

14.García G.,Francisco J,Larrión Z., José L, Rodríguez M.,Leocadio. (2011) Fragilidad: un fenotipo en revisión. GacSanit. 2011; 25(S):51-58 
15. Acosta B., Miguel Á., (2017). Dependencia en el anciano frágil: aspectos médico legales y deontológicos. Tesis doctoral. Universidad complutense de Madrid, Facultad de Medicina. Disponible en: http://eprints.ucm.es/41271/1/T38409.pdf

16. Ingles de la Torre, Marta, (2014). Identificación de biomarcadores de fragilidad en el estudio de Toledo de envejecimiento saludable .Tesis doctoral. Universidad de Valencia, Facultad de fisioterapia. Disponible en: https://dialnet.unirioja.es/institucion/uv/tesis;jsessionid=1E985544537893DD1A24 60D1F218A818.dialnet02?tipo busqueda=INSTITUCIONYTEXTOCOMPLETO\& clave busqueda $=819118 \&$ inicio $=3741$

17.Rodríguez J.,Klara S., Reales Ch.,Lisbeth J. (2016). Síndrome de fragilidad y sus variables asociadas. Artículo de revisión. Rev. Med Interna (Caracas) 2016; $32 \quad$ (4): $272 \quad$ - $278 . \quad$ Disponible en:www.svmi.web.ve/ojs/index.php/medint/article/download/396/389

18. García J., Barrera I. (2011), El Síndrome de Fragilidad un estado crónico de la vida.Boletín UISESS (Boletín de la Unidad de Investigación Social, Epidemiología y en Servicios de Salud). Vol.2 Núm. 2. Págs. 8 -10. Disponible en:https://dialnet.unirioja.es/servlet/articulo?codigo=3877170

19. Carrasco G, Marcela. Fragilidad: Un síndrome geriátrico en evolución Escuela de medicina. Facultad de medicina. Pontificia universidad católica de chile Disponible en: https://medicina.uc.cl/publicacion/fragilidad-sindrome-geriatricoevolucion

20.Sánchez Pedro. (2013). Prevalencia y atributos de la fragilidad en una cohorte española mayor de 70 años. Tesis Doctoral. Universidad de castilla- La mancha. Facultad de Medicina de Albacete. Disponible en: https://ruidera.uclm.es/xmlui/bitstream/handle/10578/3262/TESIS\%20S\%C3\%A1 nchez\%20Jurado.pdf?sequence $=1$

21.González M.,Alina M., Rodríguez R., Liliams. (2008). Fragilidad, paradigma de la atención al adulto mayor."GEROINFO. RNPS. 2110. Vol. 3 No. 1. 2008 Disponible en: $\underline{\text { http://www.sld.cu/galerias/pdf/sitios/gericuba/fragilidad.pdf }}$

22. Walston J, Fried LP. (1999).Frailty and the older man. Med. Clin. North.Am. 1999; 83:1173-94. 8.

23. Fried LP, Tangen CM, Walston J, et al.(2001). Frailty in older adults: evidence for a phenotype. J Gerontol. A Biol. Sci. Med Sci. 2001;56:M146-56. 


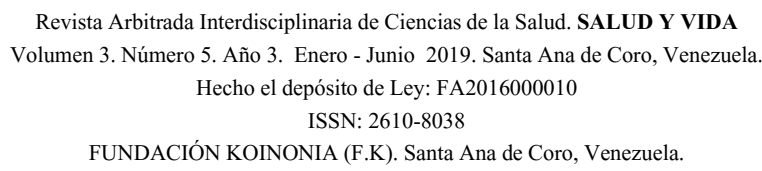

Milagros García; Moreno Martha

24.Xue QL. (2011)The frailty syndrome: definition and natural history. ClinGeriatr Med.2011;27:1-15.

25. Abizanda P, López Torres J, Romero L, López M, Sánchez PM, Atienzar P. Fragilidad y dependencia en Albacete (estudio FRADEA): Razonamiento, diseño y metodología. Rev Española Geriatría y Gerontología 2011; 46 (2): 81-8. Disponible en: http://www.elsevier.es/es-revista-revista-espanola-geriatriagerontologia-124-articulo-fragilidad-dependencia-albacete-estudio-fradea-$\underline{\text { S0211139X10002544 }}$

26. García-García FJ GAG, Alfaro Acha A, et al. The prevalence of frailty syndrome in an older population from Spain. The Toledo StudyforHealthyAging. JNHA. 2011. DOI: 10.1007/S12603-011-0075-8.

27.Santos-Eggimann B, Cuenoud P, Spagnoli J, et al. (2009). Prevalence of frailty in middle-aged and older community-dwelling Europeans living in 10 countries. J Gerontol A BiolSci Med Sci. 2009;64:675-81.

28. Alvarado BE, Zunzunegui MV, Beland F, et al.(2008). ife course social and health conditions linked to frailty in Latin American older men and women. J Gerontol A BiolSci Med Sci. 2008;63:1399-406.

29. Ottenbacher KJ, Graham JE, Al Snih S, et al. Mexican Americans and frailty: findings from the Hispanic established populations epidemiologic studies of the elderly. Am J Public Health. 2009;99:673-9.

30. Rivadeneyra Espinoza L, Sánchez Hernández C. (2016). Síndrome de fragilidad en el adulto mayor en una comunidad rural de Puebla, México. RevDuazary 2016; $13 \quad$ (2): $119-25 . \quad$ Disponible en: https://biblat.unam.mx/es/revista/duazary/articulo/sindrome-de-fragilidad-en-eladulto-mayor-en-una-comunidad-rural-de-puebla-mexico

31.Yábar-P., Cecilia,Ramos Willy,Rodríguez-Casamayor,Luis,Díaz-Santisteban Víctor. (2016). Prevalencia, características clínicas, socio- familiares y factores asociados a fragilidad en adultos mayores de 75 años de un hospital de Chimbote. Octubre 2006 - abril 2007. Disponible en: https://www.researchgate.net/publication/239570855 Prevalencia caracteristicas clinicas sociofamiliares y factores asociados a fragilidad en adultos mayor es de 75 anos de un hospital de Chimbote Octubre 2006 - abril 2007

32. Moreno P., Mujica M., Puerta M.(2013) Síndrome de fragilidad en adulto a mayores de Cabure, Municipio Petit, Estado Falcón. Enero Abril - 2013. Trabajo 


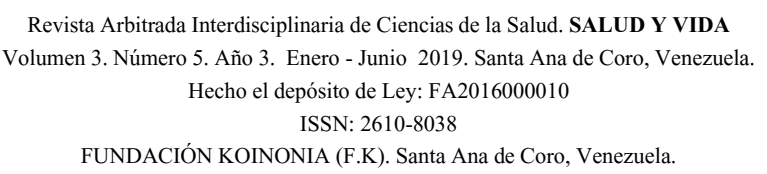

Milagros García; Moreno Martha

Especial de Grado para optar al Título de Médico Cirujano - UNEFM 2013. No publicado.

33. Bravo M., Adelmary, García, Milagros (2017). Síndrome de fragilidad y factores asociados en el adulto mayor asistido en el primer nivel de atención médica. 2017. Trabajo especial de grado para optar al título de especialista en medicina Familiar- UNEFM. No publicado.

34.Correo del Orinoco. 24 Febrero, 2012. Venezuela atraviesa una etapa de transición demográfica.

Disponible en: http://www.correodelorinoco.gob.ve/venezuela-atraviesa-una-etapa-transiciondemografica/.

35. Haiman El Troudi. (2018). Venezuela en plena Transición Demográfica. Disponible en: https://www.aporrea.org/economia/a265911.htm.

36. Camba Trujillo Nelson E., Flores Betancourt Rafael G.(2010). Protección social al adulto mayor: un análisis del ordenamiento jurídico venezolano. Gaceta Laboral v.16 n.1 Maracaibo abr. 2010 Disponible en: http://www.scielo.org.ve/scielo.php?script=sci arttext\&pid=S1315$\underline{85972010000100004 .}$

37. Instituto Nacional de Estadísticas (INE). Reporte Sociodemográfico - 2008. República Bolivariana de Venezuela. Disponible en: www.ine.gov.ve.

38. Davies, Vanesa. (2017). La migración de jóvenes hace que la población de Venezuela esté cada vez más envejecida. Viernes, 13 de octubre de 2017. El Nacional. Disponible en: http://contrapunto.com/noticia/la-migracion-de-joveneshace-que-la-poblacion-de-venezuela-envejezca-cada-vez-mas-164571/

39. Freitez L.Anitza. Venezuela: transición y riesgos socio-demográficos. Disponible en: http://gumilla.org/biblioteca/bases/biblo/texto/SIC2013760 498-501.pdf

40.De Lima, Blanca. (2003). Venezuela: envejecer en la pobreza. Disponible en: https://www.researchgate.net/publication/273451398_Venezuela_envejecer_en_I a_pobreza [consultado Nov 20 2018].

41.Bermúdez Gil L, Jiménez Julio C, Roa Adarraga L, Santana Suárez M, Quintero MV. Índice de fragilidad en el adulto mayor del Hogar Madre Marcelina. Rev Salud en Movimiento 2015; 7 (1): 0-0. Disponible en: http://publicaciones.unisimonbolivar.edu.co/rdigital/ojs/index.php/saludmov/article /view/1338. 


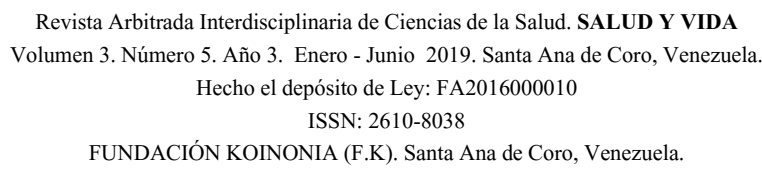

Milagros García; Moreno Martha

42. Fontecha J. (2013). Sistema móvil para la detección y valoración del síndrome de fragilidad en el adulto mayor. Repositorio Universidad de Recursos Abiertos 2013. Disponible en: http://hdl.handle.net/10578/3957.

43.OPS. (2002). La salud y el envejecimiento. 130.va Sesión del Comité Ejecutivo Washington, D.C., EUA, 24-28 de junio de 2002. Disponible en: http://iris.paho.org/xmlui/bitstream/handle/123456789/21469/ce130-15s.pdf? sequence $=1$ \&isAllowed $=y$

44.Árraga Barrios Marisela Virginia. (2011). Seguridad social para adultos mayores en Venezuela: características, bondades y limitaciones del Régimen Prestacional de Servicios Sociales al Adulto Mayor y Otras Categorías de Personas. Revista Gaceta Laboral Vol. 17, No. 2 (2011): 191 - 211 Universidad del Zulia (LUZ) • ISSN 1315-859 Disponible en: http://www.produccioncientifica.luz.edu.ve/index.php/gaceta/article/viewFile/3806/ 3805.

45.ISAGS (instituto suramericano de gobierno en salud) -UNASUR. (2017). Desafíos del Envejecimiento poblacional para los sistemas de salud en Suramérica. Hacia el envejecimiento saludable. Diciembre 2017 Disponible en: http://isags-unasur.org/wp-content/uploads/2018/04/tdr4-sus-desafios-delenvejecimiento-poblacional-para-los-sistemas-de-salud-en-suramerica-hacia-elenvejecimiento-salud-en-suramerica-hacia-el-envejecimiento-digital-22-03-1.pdf

46. Contreras, Lisbeth; y Chipia, Joan. (2016). Envejecimiento: un reto para la salud pública. Mérida-Venezuela. Revista Gicos 1(2):17-35, 2016 Disponible en: file://C:/Users/Milagro/Downloads/2016-Envejecimiento-GICOS.pdf consultado $12 / 10$.

47. Nezer de Landaeta Isis. (2009). El envejecimiento: aspectos bioéticos, políticos, económicos y sociales GacMéd Caracas. v.117 n.2 Caracas jun. 2009 Disponible en: $\quad$ http://www.scielo.org.ve/scielo.php?script=sci arttext\&pid=S0367$\underline{47622009000200007 .}$

48. Organización Mundial de la Salud. (2002). Envejecimiento activo: un marco político. RevEspGeriatrGerontol 2002; 37(S2):74-105 Disponible en: https://ccp.ucr.ac.cr/bvp/pdf/vejez/oms envejecimiento activo.pdf.

49. Villarreal R.Enrique, Paredes C.,Alba G.,Vargas D.,Emma R., Galicia R., Liliana, Martínez G., Lidia, Hernández M., Fabiola.(2015). Costo de la atención médica de pacientes con síndrome de fragilidad vs. pacientes con pre-fragilidad. Rev Cubana Med Gen Integr vol.31 no.1 Ciudad de La Habana ene.-mar. 2015. 


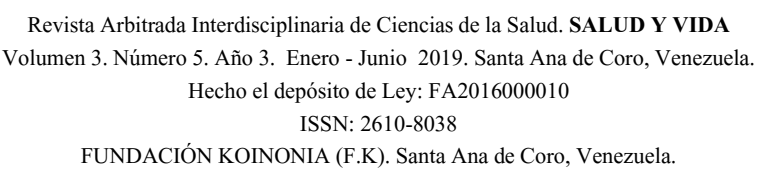

Milagros García; Moreno Martha

50.Confederación española de Alzheimer (CEAFA). (2013). La fragilidad debe ser una prioridad en el diseño de políticas de atención sanitaria a los mayores. 19 de junio de 2013. Disponible en: https://www.ceafa.es/es/quecomunicamos/noticias/la-fragilidad-debe-ser-una-prioridad-en-el-diseno-depoliticas-de-atencion-sanitaria-a-los-mayores.

51.Romero Cabrera Ángel Julio. (2010). Fragilidad un síndrome geriátrico emergente. Revista Electrónica de las Ciencias Médicas en Cienfuegos ISSN: 1727-897X Medisur 2010; 8(6) emerginggeriatric síndrome. Disponible en: http://www.redalyc.org/html/1800/180019804014/.

52.PavarinilSofia C.; Barhall Elizabeth J.; Zazzetta de Mendiondolll Marisa; Alves F. Carmen;PetrilliJosé F., †; Dos Santos Ariene. (2009). La familia y la vulnerabilidad social: un estudio con octogenarios Rev. Latino-Am. Enfermagem vol.17 no.3 RibeirãoPretoMay/June 2009 . Disponible en: http://www.scielo.br/scielo.php?pid=S0104$11692009000300015 \&$ script=sci_arttext\&tlng=es .

53. FernándezCarlos francisco.(2018) En el país, cada vez más viejos y..desprotegidos. El Tiempo. Dispoible en: https://www.eltiempo.com/vida/salud/panorama-de-los-adultos-mayores-encolombia-a-2018-213710.

54.Tamez V., Blanca M.,Ribeiro F., Manuel. (2012). El proceso de envejecimiento y su impacto socio-familiar. rev.latinoam.estud.fam. Vol. 4, enero - diciembre, 2012. $\begin{array}{llllll}\text { pp. } & 11 & - & 30 & \text { Disponible }\end{array}$ http://revlatinofamilia.ucaldas.edu.co/downloads/Rlef4 2.pdf

55. Rojas Jarquín Diana. (2014). "Tipología y funcionalidad familiar en el adulto mayor con síndrome de fragilidad que acude a la UMF 220, Toluca, México de diciembre del 2012 a junio del 2013." Tesis que para obtener el diploma de la especialidad de medicina familiar.Disponible en: http://ri.uaemex.mx/handle/20.500.11799/14573.

C2019 por los autores. Este artículo es de acceso abierto y distribuido según los términos y condiciones de la licencia Creative Commons Atribución-NoComercial-Compartirlgual 4.0 Internacional (CC BY-NC-

SA 4.0) (https://creativecommons.org/licenses/by-nc-sa/4.0/). 